\title{
The empirical Gaia G-band extinction coefficient
}

\author{
C. Danielski ${ }^{1,2}$, C. Babusiaux ${ }^{1,3}$, L. Ruiz-Dern ${ }^{1}$, P. Sartoretti ${ }^{1}$, and F. Arenou ${ }^{1}$ \\ ${ }^{1}$ GEPI, Observatoire de Paris, PSL Research University, CNRS, 5 Place Jules Janssen, 92190 Meudon, France \\ e-mail: camilla.danielski@obspm.fr \\ 2 Institut d'Astrophysique de Paris, CNRS, UMR 7095, Sorbonne Université, 98 bis boulevard Arago, 75014 Paris, France \\ ${ }^{3}$ Université Grenoble-Alpes, CNRS, IPAG, 38000 Grenoble, France
}

Received 20 November 2017 / Accepted 5 February 2018

\begin{abstract}
Context. The first Gaia data release unlocked the access to photometric information for 1.1 billion sources in the $G$-band. Yet, given the high level of degeneracy between extinction and spectral energy distribution for large passbands such as the Gaia G-band, a correction for the interstellar reddening is needed in order to exploit Gaia data.

Aims. The purpose of this manuscript is to provide the empirical estimation of the Gaia $G$-band extinction coefficient $\mathrm{k}_{G}$ for both the red giants and main sequence stars in order to be able to exploit the first data release DR1.

Methods. We selected two samples of single stars: one for the red giants and one for the main sequence. Both samples are the result of a cross-match between Gaia DR1 and 2MASS catalogues; they consist of high-quality photometry in the $G$-, $J$ - and $K_{\mathrm{S}}$-bands. These samples were complemented by temperature and metallicity information retrieved from APOGEE DR13 and LAMOST DR2 surveys, respectively. We implemented a Markov chain Monte Carlo method where we used $\left(G-K_{\mathrm{S}}\right)_{0}$ versus $T_{\text {eff }}$ and $\left(J-K_{\mathrm{S}}\right)_{0}$ versus $\left(G-K_{\mathrm{S}}\right)_{0}$ calibration relations to estimate the extinction coefficient $\mathrm{k}_{G}$ and we quantify its corresponding confidence interval via bootstrap resampling. We tested our method on samples of red giants and main sequence stars, finding consistent solutions.

Results. We present here the determination of the Gaia extinction coefficient through a completely empirical method. Furthermore we provide the scientific community with a formula for measuring the extinction coefficient as a function of stellar effective temperature, the intrinsic colour $\left(G-K_{\mathrm{S}}\right)_{0}$, and absorption.
\end{abstract}

Key words. methods: data analysis - methods: statistical - techniques: photometric - dust, extinction stars: fundamental parameters

\section{Introduction}

When it comes to understanding the physics of disk galaxies, our location within the Milky Way plays an important role. By observing our visible sky and studying the astrophysical processes of its individual components, we can learn about the structure and dynamics of the Galaxy, and hence infer its formation and evolution. This prospect would not be possible only by examining other galaxies.

Accordingly, numerous spectro/photometric surveys have been conducted over the last decade, altogether spanning different spectral ranges to cover a wide variety of galactic astrophysical processes. To mention some: the Fermi Gamma-ray space Telescope (GLAST; Atwood et al. 2009) in the gammaray range, XMM-Newton (Mason et al. 2001; Rosen et al. 2016) in the X-ray, the Galaxy Evolution Explorer (GALEX; Martin et al. 2005) in the ultraviolet (UV), the Sloan Digital Sky Survey (SDSS; York et al. 2000) in the optical, the two-micron All-Sky Survey (2MASS; Skrutskie et al. 2006) in the near infrared (NIR), and Planck (Planck Collaboration I 2011) in the far infrared-microwave range.

Yet, in the midst of all these surveys, the mapping process of the Milky Way is culminating with Gaia, the ESA space mission that has just started providing data to study formation, dynamical, chemical and star-formation evolution (Perryman et al. 2001; Gaia Collaboration 2016). Nonetheless, despite the unrivalled completeness of its information, Gaia, like the other surveys, does not rule out astrophysical selection effects such as interstellar extinction.
Extinction is caused by the presence of dust in the line of sight and it has the main effect of dimming sources and reddening them. In particular, around $30 \%$ of light in the UV, optical, and NIR is scattered and absorbed due to the interstellar medium (Draine 2003). In broad-band photometry, an additional major hurdle to face is the substantial degeneracy between extinction, effective temperature $T_{\text {eff }}$, and spectral energy distribution (SED). This degeneracy limits the accuracy with which any of the parameters can be estimated (Bailer-Jones 2010). It is important to mention that extinction coefficients $\mathrm{k}_{\lambda}$ are a function of wavelength and become greater towards shorter wavelengths; they are defined as $\mathrm{k}_{\lambda}=A_{\lambda} / A_{\text {ref }}$ where $A_{\lambda}$ is the absolute absorption at any wavelength, expressed relative to the absolute absorption at a chosen reference wavelength $A_{\text {ref }}$ (Cardelli et al. 1989).

Over recent years an increased number of studies have focused on delivering more precise extinction coefficient values for various known pass-bands by using a combination of spectroscopic and photometric information retrieved from the most advanced surveys (e.g. Yuan et al. 2013; Schlafly et al. 2016; Xue et al. 2016).

Important to note, however, is that in the case of a wide passband, like the Gaia one, a star which has the greater fraction of its radiation in the blue end of the spectrum (a bluer star), has a larger extinction coefficient than a redder star. Exact knowledge of the passband is therefore essential to correctly estimate the reddening factor.

Reddening of an object in a given colour can be described by the colour excess which is the difference between its observed 


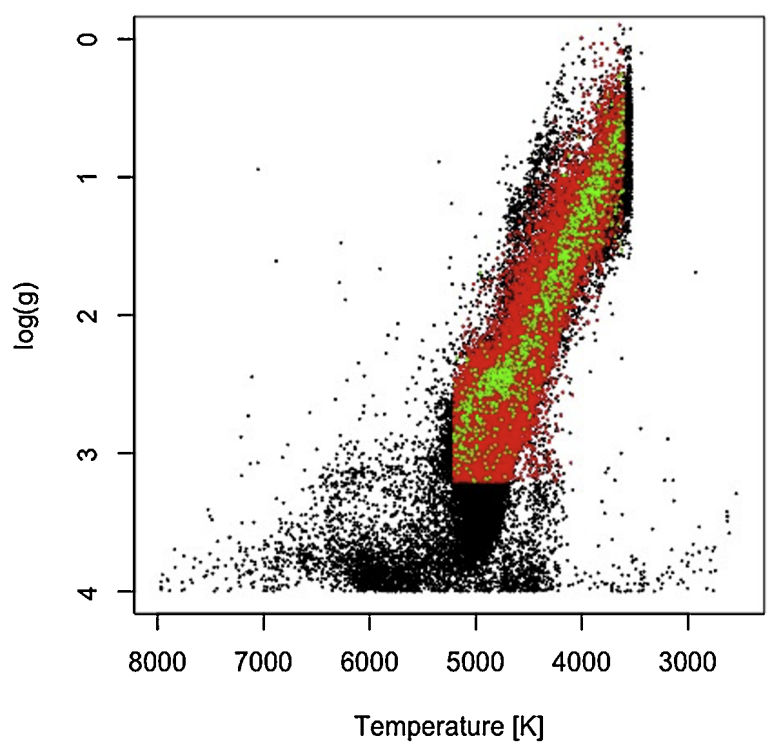

colour and its intrinsic value. For instance, the colour excess between the Gaia $G$-band and the 2MASS $K_{\mathrm{S}}$-band is given by $\mathrm{E}\left(G-K_{\mathrm{S}}\right)=\left(G-K_{\mathrm{S}}\right)_{\text {obs }}-\left(G-K_{\mathrm{S}}\right)_{0}$, where $\left(G-K_{\mathrm{S}}\right)_{\text {obs }}$ is the observed colour and $\left(G-K_{\mathrm{S}}\right)_{0}$ is the intrinsic one.

At the time of the publication of Gaia DR1, only the nominal Gaia $G$-passband, modelled with the most up-to-date pre-launch information, was available (Jordi et al. 2010). Recently a calibration of the Gaia $G$-DR1 passband has been provided by Maíz Apellániz (2017). The second is redder than the first one due to some water contamination in the optics, which diminished the spectral efficiency more in the blue part of the band than in the red part (Gaia Collaboration 2016). A new filter response curve will be available with the second Gaia data release (DR2). Uncertainties, either in the passband determination or in the extinction law or in the stellar model atmospheres, can yield to inaccurate extinction coefficients. For these reasons and because the accurate determination of reddening to a star is key for exploiting the available Gaia data, we present here a determination of Gaia extinction coefficient for both red giants and dwarfs stars through a completely empirical method.

The manuscript is structured as follows. Section 2 introduces the data we used and describes the data selection for the red giants and dwarfs sample, respectively. In Sect. 3 we estimate the photometric calibration relations for the main sequence. Section 4 describes the estimation of the theoretical extinction coefficients used in our analysis. In Sect. 5 we present the technique we used to estimate Gaia $G$-band extinction coefficient $\left(\mathrm{k}_{G}\right)$ for the red giants sample, the dwarfs sample, and, finally, for the union of both samples. In Sect. 6 we present the results and discuss them. Finally, Sect. 7 presents our conclusions.

\section{Data}

For our analysis we cross-matched photometric and spectroscopic data from different surveys. More specifically, for the photometric information we used the Gaia DR1 and 2MASS catalogues. The $2 \mathrm{MASS} J, H, K_{\mathrm{S}}$ magnitudes are available for a large fraction of the Gaia sources and the near-infrared extinction law is fairly well characterized (e.g. Fitzpatrick \& Massa 2009).

Spectroscopic parameters, such as effective temperature $T_{\text {eff }}$, surface gravity $\log (g)$, and metallicity $[\mathrm{Fe} / \mathrm{H}]$, were retrieved from surveys selected ad hoc for the samples analysed. Our

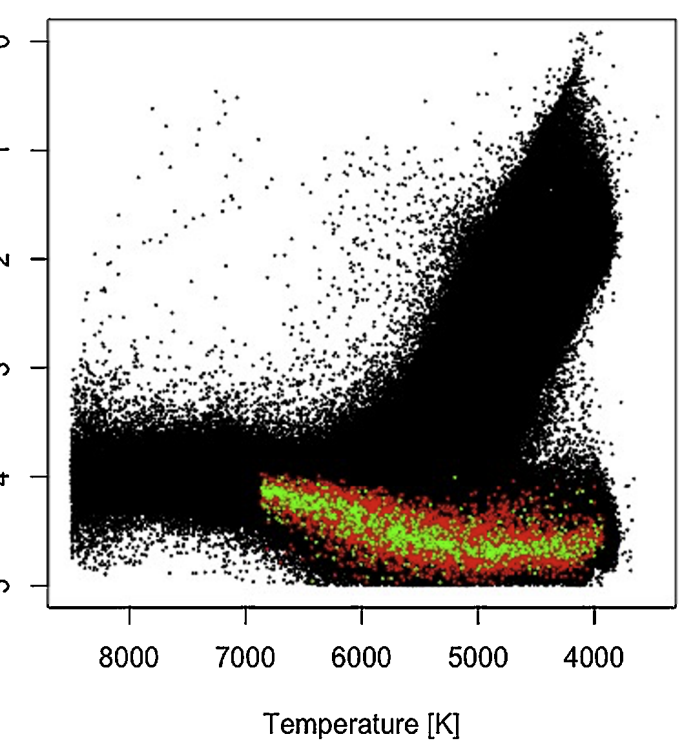

Fig. 1. Data selection: spectroscopic surveys used for the red giants (APOGEE stars - data release 13, left) and the main sequence (LAMOST stars data release 2, right) analysis. Black points represent stars in each data release, red points are objects in the first sample selection, used to differentiate red giant stars from dwarfs and vice versa. Green points represent a 1000 star sample selection we used to measure the extinction coefficient.

analysis was performed on both the red giants (RG) sample and the main sequence (MS) sample (Fig. 1) separately, then on both samples combined together.

\subsection{The red giant sample}

Effective temperature $T_{\text {eff }}$, surface gravity $\log (g)$, and metallicity $[\mathrm{Fe} / \mathrm{H}]$ were retrieved from the spectroscopic survey APO Galactic Evolution Experiment (APOGEE), DR13 (Albareti et al. 2017).

The cross-match between APOGEE and Gaia was done using the 2MASS ID provided in APOGEE and the 2MASSGDR1 cross-matched catalogue (Marrese et al. 2017), where we kept only cross-matched stars with angular distance lower than $0.3^{\prime \prime}$. Hence, we selected those stars with high infrared photometric quality (i.e. 2MASS "AAA" quality flag), radial velocity error $\sigma_{\mathrm{RV}}<0.1 \mathrm{~km} \mathrm{~s}^{-1}$ to exclude binary stars, and photometric errors of $\sigma_{G}<0.01 \mathrm{mag}, \sigma_{J}<0.03 \mathrm{mag}$ and $\sigma_{K_{\mathrm{S}}}<0.03 \mathrm{mag}$. The $G$-band photometric error was later increased by $0.01 \mathrm{mag}$ in quadrature to mitigate the impact of bright stars' residual systematics (Evans et al. 2017; Arenou et al. 2017). We then retained the red giants stars by screening those with colour $\left(G-K_{\mathrm{S}}\right)_{\text {obs }}>1.6 \mathrm{mag}$. For stars with parallax information in Gaia DR1 (TGAS), we used the same criteria as Ruiz-Dern et al. (2018):

$$
G+5+5 \log _{10}\left(\frac{\varpi+2.32 \sigma_{\varpi}}{1000}\right)<4
$$

where the factor 2.32 on the parallax error $\sigma_{\varpi}$ corresponds to the 99th percentile of the parallax probability density function. When no parallax information was available, the selection was performed by filtering on the surface gravity $(\log (g)<3.2 \mathrm{dex})$. Finally, we selected those stars with effective temperature $3603 \mathrm{~K}<T_{\text {eff }} \pm \sigma_{\text {Teff }}<5207 \mathrm{~K}$ and metallicity $-1.5 \mathrm{dex}<[\mathrm{Fe} / \mathrm{H}]<0.4 \mathrm{dex}$ to work within the same limits set for the $T_{\text {eff }}$ versus $\left(G-K_{\mathrm{S}}\right)_{0}$ calibration by Ruiz-Dern et al. (2018). The application of these criteria delivered a sample of 71290 stars.

\subsection{The main sequence sample}

For the dwarfs sample we cross-matched our photometric samples with the Large sky Area Multi-Object fiber Spectroscopic Telescope survey (LAMOST; Zhao et al. 2012) DR2, 
Table 1. Coefficients of the calibration relations (Eq. (1)) and their uncertainties for the RG sample (Ruiz-Dern et al. 2018) and MS sample (this work).

\begin{tabular}{lcccccc}
\hline \hline & RMS & $c_{1}$ & $c_{2}$ & $c_{3}$ & $c_{4}$ & $c_{5}$ \\
\hline RG & 0.05 & $13.554 \pm 0.478$ & $-20.429 \pm 1.020$ & $8.719 \pm 0.545$ & $0.143 \pm 0.013$ & $-0.0002 \pm 0.009$ \\
MS & 0.04 & $6.946 \pm 0.181$ & $-6.835 \pm 0.354$ & $1.711 \pm 0.172$ & - & - \\
\hline & & $c_{7}$ & $c_{8}$ & $c_{9}$ & $c_{10}$ & - \\
\hline RG & 0.02 & $-0.227 \pm 0.024$ & $0.466 \pm 0.021$ & $-0.023 \pm 0.005$ & $-0.016 \pm 0.002$ & $-0.005 \pm 0.001$ \\
MS & 0.02 & $-0.200 \pm 0.034$ & $0.471 \pm 0.038$ & $-0.03 \pm 0.01$ & - & - \\
\hline
\end{tabular}

Notes. The RMS corresponds to one standard deviation of the relations residuals.

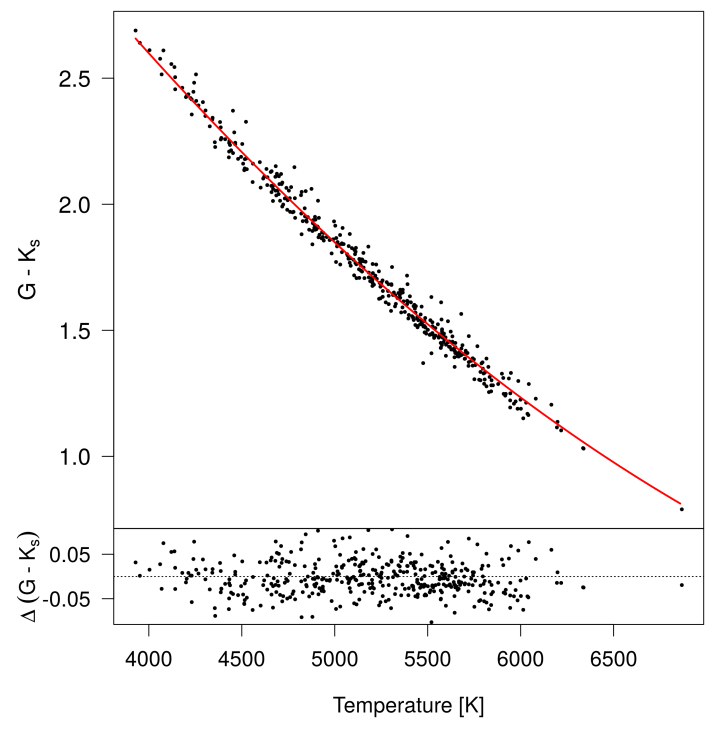

from which we retrieved effective temperature $T_{\text {eff }}$, surface gravity $\log (g)$, and metallicity $[\mathrm{Fe} / \mathrm{H}]$ (Wu et al. 2014). The cross-match with 2MASS and Gaia DR1 was done with a radius of $0.2^{\prime \prime}$. We selected a sub-sample of objects with radial velocity error $\sigma_{\mathrm{RV}}<20 \mathrm{~km} \mathrm{~s}^{-1}$ to exclude binary stars, photometric errors $\sigma_{\mathrm{G}}, \sigma_{\mathrm{K}}, \sigma_{\mathrm{J}}<0.03 \mathrm{mag}$ and relative temperature error smaller than 5\%. As explained in Sect. 2.1, we increased $\sigma_{\mathrm{G}}$ of $0.01 \mathrm{mag}$ in quadrature. Following this we retained the main sequence stars by applying a surface gravity cut:

$\log (g)-2 \sigma_{\log (g)}>3.5$ dex,

where $\sigma_{\log (g)}$ is the surface gravity error.

We set the metallicity range for the MS calibration (and consequently for the extinction coefficient estimation) to be solar-like $(-0.05 \mathrm{dex}<[\mathrm{Fe} / \mathrm{H}]<0.05 \mathrm{dex})$ because of the significant correlation between metallicity and effective temperature in the LAMOST data which did not allow a good convergence of the photometric calibration (see Sect. 3).

We further selected stars with temperature within the calibration temperature interval $\left(3928 \mathrm{~K}<T_{\text {eff }} \pm \sigma_{\text {Teff }}<6866 \mathrm{~K}\right)$, leaving a final sample of 17468 dwarfs.

\section{Photometric calibration}

In order to empirically measure the Gaia $G$-band extinction coefficient $\mathrm{k}_{G}$, the colour excess $\mathrm{E}\left(G-K_{\mathrm{S}}\right)$ and $\mathrm{E}\left(J-K_{\mathrm{S}}\right)$ for our samples need to be determined. To do so we used for the RG sample the photometric calibration relations presented in Ruiz-Dern et al. (2018) while, for the MS sample, we applied the method described therein to empirically retrieve the photometric calibration relations. Specifically, the calibration relations for both samples were modelled as the following:

$$
\begin{aligned}
\left(G-K_{\mathrm{S}}\right)_{0}= & c_{1}+c_{2} \hat{T}+c_{3} \hat{T}^{2}+c_{4}[\mathrm{Fe} / \mathrm{H}]+\ldots \\
& +c_{5}[\mathrm{Fe} / \mathrm{H}]^{2}+c_{6} \hat{T}[\mathrm{Fe} / \mathrm{H}]^{2} \\
\left(J-K_{\mathrm{S}}\right)_{0}= & c_{7}+c_{8}\left(G-K_{\mathrm{S}}\right)_{0}+c_{9}\left(G-K_{\mathrm{S}}\right)_{0}^{2}+\ldots \\
& +c_{10}[\mathrm{Fe} / \mathrm{H}]+c_{11}[\mathrm{Fe} / \mathrm{H}]^{2}+\ldots \\
& c_{12}\left(G-K_{\mathrm{S}}\right)_{0}[\mathrm{Fe} / \mathrm{H}]
\end{aligned}
$$

where $\hat{T}=T_{\text {eff }} / 5040$ is the normalised temperature and $c_{i}$ are the coefficients reported in Table 1 for both RG and MS samples.

For calibrating the main sequence relations we selected from the sample of Sect. 2.2 only low extinction stars $(\mathrm{E}(B-V)<0.01)$ selected from the recent three-dimensional (3D) local extinction map of Capitanio et al. (2017) or the twodimensional (2D) map of Schlegel et al. (1998) when no distance information was available. We required the relative temperature error to be smaller than $2 \%$. The application of these further criteria left a total of 415 stars that we used for the calibration process. Please refer to Ruiz-Dern et al. (2018) for more details on the calibration method. Figure 2 shows the relations (Eq. (1), Table 1) which were established within the interval of temperature [3928 K, $6866 \mathrm{~K}$ ].

\section{Theoretical extinction coefficients}

We computed the theoretical extinction coefficients $k_{m}$ using the Fitzpatrick \& Massa (2007) extinction law $E_{\lambda}$, the Kurucz 
Table 2. Theoretical extinction coefficients for the Gaia G-band, both pre-launch (Jordi et al. 2010) and G-DR1 (Maíz Apellániz 2017) passbands, and for $J$ - and $K_{\mathrm{S}}$-bands (see Sect. 4).

\begin{tabular}{|c|c|c|c|c|c|c|c|}
\hline $\mathrm{RG}+\mathrm{MS}$ & $a_{1}$ & $a_{2}$ & $a_{3}$ & $a_{4}$ & $a_{5}$ & $a_{6}$ & $a_{7}$ \\
\hline $\mathrm{k}_{G}\left(\hat{T}, A_{0}\right)$ & -0.317797257 & 2.538901003 & -1.997742387 & 0.572289388 & -0.013179503 & 0.000607315 & -0.01126344 \\
\hline $\mathrm{k}_{G}\left(\hat{T}, A_{0}\right)-\mathrm{DR} 1$ & -0.279133556 & 2.373624663 & -1.878795709 & 0.53904796 & -0.011673326 & 0.000544945 & -0.010233727 \\
\hline $\mathrm{k}_{J}\left(\hat{T}, A_{0}\right)$ & 0.252033852 & -0.042526876 & 0.044560182 & -0.013883035 & -0.000239872 & $8.45 \mathrm{E}-07$ & $-1.96 \mathrm{E}-05$ \\
\hline $\mathrm{k}_{K_{\mathrm{S}}}\left(\hat{T}, A_{0}\right)$ & 0.073839341 & 0.03381806 & -0.03063728 & 0.009124118 & $-1.95 \mathrm{E}-05$ & $9.66 \mathrm{E}-09$ & $-6.81 \mathrm{E}-07$ \\
\hline $\mathrm{k}_{G}\left(\left(G-K_{\mathrm{S}}\right)_{0}, A_{0}\right)$ & 0.935556283 & -0.090722012 & 0.014422056 & -0.002659072 & -0.030029634 & 0.000607315 & 0.002713748 \\
\hline $\mathrm{k}_{J}\left(\left(G-K_{\mathrm{S}}\right)_{0}, A_{0}\right)$ & 0.242998 & -0.00 & 0.000107601 & 2.54 & -0.00 & $8.45 \mathrm{E}-07$ & $4.60 \mathrm{E}-06$ \\
\hline $\mathrm{k}_{K_{\mathrm{S}}}\left(\left(G-K_{\mathrm{S}}\right)_{0}, A_{0}\right)$ & 0.086033161 & $7.65 \mathrm{E}-05$ & $8.54 \mathrm{E}-06$ & $-1.52 \mathrm{E}-05$ & $-2.00 \mathrm{E}-05$ & - & - \\
\hline $\mathrm{k}_{G}\left(\left(G-K_{\mathrm{S}}\right)_{0}, A_{0}\right)-\mathrm{DR} 1$ & 0.882095056 & -0.086780236 & 0.01511573 & -0.002963829 & -0.027054718 & 0.000544945 & 0.002604135 \\
\hline $\mathrm{k}_{J}\left(\left(G-K_{\mathrm{S}}\right)_{0}, A_{0}\right)-\mathrm{DR} 1$ & 0.243062354 & -0.001899476 & 0.000140615 & $2.58 \mathrm{E}-05$ & -0.000269124 & $8.45 \mathrm{E}-07$ & $4.86 \mathrm{E}-06$ \\
\hline $\mathrm{k}_{K_{\mathrm{S}}}\left(\left(G-K_{\mathrm{S}}\right)_{0}, A_{0}\right)-\mathrm{DR} 1$ & 0.086025432 & $9.23 \mathrm{E}-05$ & $3.32 \mathrm{E}-06$ & $-1.65 \mathrm{E}-05$ & $-2.00 \mathrm{E}-05$ & - & - \\
\hline
\end{tabular}

Notes. The extinction coefficients are modelled as function of $\left(\hat{T}, A_{0}\right)$ and $\left(\left(G-K_{\mathrm{S}}\right)_{0}, A_{0}\right)$, Eq. (3), and they are valid for both the red giants and the main sequence samples for $3500 \mathrm{~K}<T_{\text {eff }}<7000 \mathrm{~K}$ and $A_{0}<20$ mag.
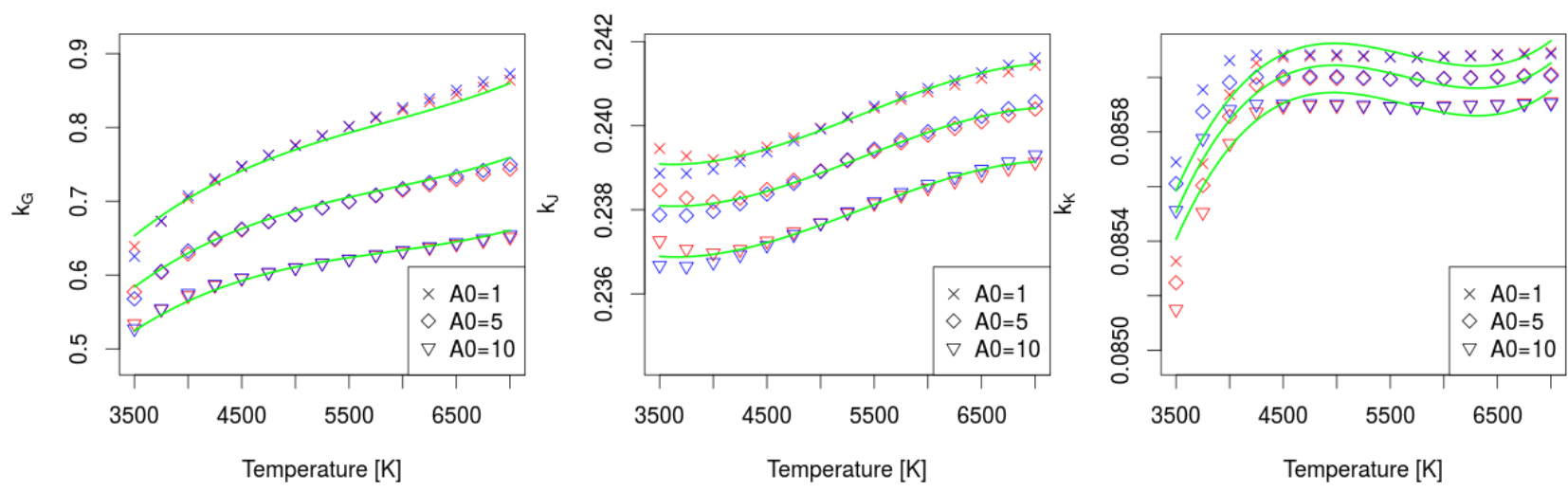

Fig. 3. Theoretical extinction coefficients in the $G$ - (Gaia), $J$ - and $K_{S^{-}}(2 \mathrm{MASS})$ bands as a function of temperature for different extinctions $\left(A_{0}=1,5,10 \mathrm{mag}\right.$ ) and different surface gravities: $\log (g)=2.5 \mathrm{dex}$ (red) and $\log (g)=4 \mathrm{dex}$ (blue). Green lines represent the global fit for the three absorption values.

spectral energy distributions $F_{\lambda}$ from Castelli \& Kurucz (2003) and the filters transmissions $T_{\lambda}$ :

$k_{m} A_{0}=A_{m}=m-m_{0}=-2.5 \log _{10}\left(\frac{\int F_{\lambda} T_{\lambda} E_{\lambda}^{A_{0}} d \lambda}{\int F_{\lambda} T_{\lambda} d \lambda}\right)$,

with $A_{0}$ being the interstellar extinction at $\lambda=550 \mathrm{~nm}$ (Gaia reference value). While the Fitzpatrick \& Massa (2007) extinction law was derived using hot stars, this extinction law was calibrated using the full star spectral energy distribution and therefore should also be valid for the lower temperature stars of our sample.

2MASS transmissions were taken from Cohen et al. $(2003)^{1}$. For comparison purposes we used the Gaia pre-launch transmission $^{2}$ and the Gaia G-DR1 transmission of Maíz Apellániz $(2017)^{3}$.

As shown by Jordi et al. (2010), in such a large band as the Gaia G-band ( 330-1050 nm), the extinction coefficient varies strongly with temperature and the extinction itself, but less

\footnotetext{
1 http://www.ipac.caltech.edu/2mass/releases/allsky/ doc/sec6_4a.html

2 https://www.cosmos.esa.int/web/gaia/

transmissionwithoriginal

3 http://cdsarc.u-strasbg.fr/viz-bin/qcat?J/

$\mathrm{A}+\mathrm{A} / 608 / \mathrm{L} 8$
}

with surface gravity and metallicity. We therefore modelled the extinction coefficients as a function of $A_{0}$ and $T_{\text {eff }}$, following the formula:

$k_{m}=a_{1}+a_{2} \mathrm{X}+a_{3} \mathrm{X}^{2}+a_{4} \mathrm{X}^{3}+a_{5} A_{0}+a_{6} A_{0}^{2}+a_{7} \mathrm{X} A_{0}$,

where $\mathrm{X}$ is either $\hat{T}=T_{\text {eff }} / 5040$ or $\left(G-K_{\mathrm{S}}\right)_{0}$, depending on whether we are analysing the extinction coefficient as a function of the normalised temperature or the colour, respectively. The parameters $a_{i}$ are the coefficients of the fit in each photometric band $m$.

Table 2 reports the coefficients $a_{i}$ for the theoretical estimation of the global $\mathrm{k}_{J}$ and $\mathrm{k}_{K_{S}}$ coefficients valid for both red giants and main sequence stars, as well as $\mathrm{k}_{G}$, which was computed by using the Gaia pre-launch modelled filter response.

The fit is performed using extinctions computed on a grid with a spacing of $250 \mathrm{~K}$ in $T_{\text {eff }}$ and 0.01 mag in $A_{0}$ with $0.01 \mathrm{mag}<\mathrm{A}_{0}<20 \mathrm{mag}$ and $3500 \mathrm{~K}<T_{\text {eff }}<7000 \mathrm{~K}$ and two surfaces gravities: $\log (g)=2.5 \mathrm{dex}$ and 4 dex. The result is shown in Fig. 3. We checked that high-order parameters in the polynomials are needed with an ANOVA test. Coefficients $a_{5}$ and $a_{6}$ are not significant only for the $K_{\mathrm{S}}$-band and for relatively low extinctions $\left(A_{0}<5 \mathrm{mag}\right)$. In particular residuals of the fit are smaller than $0.3 \%$ for $\mathrm{k}_{K_{\mathrm{S}}}, 0.2 \%$ for $\mathrm{k}_{J}$ and $4.5 \%$ for $\mathrm{k}_{G}$. For $\mathrm{k}_{G}$, residuals decrease to $2.4 \%$ when the fit is performed just for $A_{0}<5$ mag. For comparison, we have estimated the extinction coefficients using the Cardelli et al. (1989) extinction law, 
and compared them to the ones obtained with the Fitzpatrick \& Massa (2007) law: the difference is of $37 \%$ in the $K_{\mathrm{S}}$-band, $20 \%$ in the $J$-band and $5 \%$ in the nominal $G$-band. Jordi et al. (2010) also assessed that $R_{V}$ variation does not have a significant impact on $\mathrm{k}_{G}$. On the other hand, the $K_{\mathrm{S}}$ - and $J$-bands are much less sensitive to spectral type variations (Fig. 3): for instance the difference between a red giant star and Vega is of only $0.07 \%$ for the $K_{\mathrm{S}}$-band and $1 \%$ for the $J$-band, while it is of $21 \%$ in the $G$-band.

\section{Method}

To empirically measure the $G$-band extinction coefficient as a function of either temperature or colour $\left(G-K_{\mathrm{S}}\right)_{0}$ we implemented a Markov-chain Monte Carlo method (MCMC, Brooks et al. 2011) to sample the parameter space and to properly account for errors. The MCMC used the jags algorithm (Plummer 2003) encompassed in runjags ${ }^{4}$ library, for $\mathrm{R}$ programme language.

In order to not affect the MCMC convergence by having an uneven distribution in extinction and temperature, we used a $2 \mathrm{D}$ kernel density estimation of the $\mathrm{E}\left(G-K_{\mathrm{S}}\right)$ versus $T_{\text {eff }}$ stellar probability space to select a more uniform sub-sample of 1000 stars for each RG and MS (Fig. 1) and combined (RG + MS) sample. The number of stars in each sub-sample (i.e. 1000) is optimised to be statistically relevant for the analysis yet without having a large disproportion of elements between bins (which could cause the analysis to be biased towards the most populated bin).

Intrinsic colours $\left(G-K_{\mathrm{S}}\right)_{0}$ and $\left(J-K_{\mathrm{S}}\right)_{0}$ were taken from Eq. (1) where temperature and metallicity were set to be $T_{\text {eff }}^{\prime} \sim \mathcal{N}\left(T_{\text {eff }}, \sigma_{\mathrm{T}_{\text {eff }}}^{2}\right)$ and $[\mathrm{Fe} / \mathrm{H}]^{\prime} \sim \mathcal{N}\left([\mathrm{Fe} / \mathrm{H}], \sigma_{[\mathrm{Fe} / \mathrm{H}]}^{2}\right)$ where $\mathcal{N}$ is the normal distribution and $\sigma_{\mathrm{Teff}}^{2}, \sigma_{[\mathrm{Fe} / \mathrm{H}]}^{2}$ the respective observed variances.

Observed colours $\left(G-K_{\mathrm{S}}\right)_{\mathrm{obs}}$ and $\left(J-K_{\mathrm{S}}\right)_{\mathrm{obs}}$ were set to be

$\left(G-K_{\mathrm{S}}\right)_{\mathrm{obs}} \sim \mathcal{N}\left(\left(G-K_{\mathrm{S}}\right)_{0}+\left(\mathrm{k}_{G}-\mathrm{k}_{K_{\mathrm{S}}}\right) \cdot A_{0}^{\prime}, \sigma_{G-K_{\mathrm{S}}}^{2}\right)$

$\left(J-K_{\mathrm{S}}\right)_{\mathrm{obs}} \sim \mathcal{N}\left(\left(J-K_{\mathrm{S}}\right)_{0}+\left(\mathrm{k}_{J}-\mathrm{k}_{K_{\mathrm{S}}}\right) \cdot A_{0}^{\prime}, \sigma_{J-K_{\mathrm{S}}}^{2}\right)$,

where $\sigma_{G-K_{\mathrm{S}}}^{2}=\left(\sigma_{G}^{2}+\sigma_{K_{\mathrm{S}}}^{2}\right)$ and $\sigma_{J-K_{\mathrm{S}}}^{2}=\left(\sigma_{J}^{2}+\sigma_{K_{\mathrm{S}}}^{2}\right)$ and where $\sigma_{G}, \sigma_{J}, \sigma_{K_{S}}$ are the photometric errors. $\mathrm{k}_{J}$ and $\mathrm{k}_{K_{S}}$ are the extinction coefficients for $J$ - and $K_{\mathrm{S}}$-bands as a function of either $\hat{T}$ or colour. All along our analysis $\mathrm{k}_{J}$ and $\mathrm{k}_{K_{\mathrm{S}}}$ are fixed to the theoretical values (see Sect. 4).

For each star in the sample we used its colour excess $\mathrm{E}\left(J-K_{\mathrm{S}}\right)$ and initial extinction coefficients values (computed at $A_{0}=0 \mathrm{mag}$ ) to get an initial value of the absorption $A_{0}$, which we then set in the MCMC as the mean of a truncated normal distribution $A_{0}^{\prime} \sim \mathcal{N}\left(A_{0}, 0.2\right)$ lying within the positive interval $A_{0}^{\prime}>0$. For a given star, its initial $A_{0}$ value does not change within the MCMC. Finally we set the coefficients $a_{i}$ of Eq. (3) free to vary following the uninformative prior distribution $a_{i} \sim \mathcal{N}(0,1000)$.

Each MCMC was run using two chains with $10^{4}$ steps and a burn-in of 4000 . We used standardised variables to improve the efficiency of MCMC sampling (hence reducing the autocorrelation in the chains) and checked for chain convergence by using the Gelman-Rubin convergence diagnostic. We tested the significance of coefficients $a_{i}$ through the Deviance Information Criterion (DIC), a model fit measure that penalises model complexity.

\footnotetext{
4 https://cran.r-project.org/web/packages/runjags/ runjags.pdf
}

We produced ten different sub-samples of 1000 stars (uniform in $T_{\text {eff }}$ and $E\left(G-K_{S}\right)$ ), each of which was processed through an MCMC. The mean of those runs is reported in Table 3.

We run this analysis for the red giants first, then for the main sequence stars, and finally for both samples combined in a single one.

Error analysis. To derive our confidence interval, we use the bootstrap resampling technique (Efron 1987). The bootstrap resampling consists of generating a large number of data sets, each with an equal amount of points randomly drawn with replacement from the original sample. It allows us to take into account not only measurement errors but also samplinginduced errors, which are here a relevant factor due to the uneven distribution of stars in temperature and colour excess space.

Bootstrapped $a_{i}$ errors are larger than MCMC chains errors by an average factor of 5,3 , and 7 for the $T_{\text {eff }}$ case and 16, 3 , and 4 for the $\left(G-K_{\mathrm{S}}\right)_{0}$ case for $\mathrm{RG}, \mathrm{MS}$, and $\mathrm{RG}+\mathrm{MS}$, respectively. Important to note though is that these uncertainties are constrained by the precision of the data used, or, more specifically, by the error on the temperature, whose median is $\tilde{\sigma}_{\text {Teff }} \sim 69 \mathrm{~K}$ for APOGEE data and $\tilde{\sigma}_{\text {Teff }} \sim 115 \mathrm{~K}$ for LAMOST data.

We carried out the MCMC runs on 100 bootstrapped samples and derived the confidence levels through the percentile method, which we report in Table 3 and Fig. 5.

\section{Results and discussion}

All MCMCs to estimate both $\mathrm{k}_{G}\left[\hat{T}, A_{0}\right]$ and $\mathrm{k}_{G}\left[\left(G-K_{\mathrm{S}}\right)_{0}, A_{0}\right]$ were found to converge for all the three samples analysed (RG, MS and RG + MS).

Table 3 reports final $a_{i}$ coefficients and their uncertainties, as well as $\mathrm{k}_{G}$ intervals of validity (i.e. temperature, colour, and extinction). The temperature interval (and consequently the colour interval) is the range common to all the bootstrapped samples employed in our analysis. The maximum extinction $\left(A_{0}\right)$ depends on the $\mathrm{E}\left(J-K_{\mathrm{S}}\right)$ data distribution. For conservative reasons, as the colour excess distribution for the three samples is right-skewed (i.e. small number of stars with large colour excess), we set the $A_{0}$ upper limit by cutting ad hoc the tail of each distribution after a visual inspection, i.e. where we had a small gap in the data or where the stars were too few to give a robust solution.

We note that, while we tested the significance of high-order $a_{i}$ parameters with the DIC test (see Sect. 5), some coefficients in Table 3 appear as non-significant due to bootstrap errors being significantly larger than the MCMC derived ones.

We show in Fig. 4 the retrieved empirical colour excess $\mathrm{E}\left(G-K_{\mathrm{S}}\right)$ versus $\mathrm{E}\left(J-K_{\mathrm{S}}\right)$ for the three samples. We picked the median of the high-extinction stars' temperature as reference temperature. For the MS sample, the median temperature does not change significantly for high-extinction stars while for the RG, the high-extinction stars are the coolest as they are intrinsically significantly brighter.

The three stellar samples delivered consistent results. We display in Fig. 5 the direct comparison of $\mathrm{k}_{G}$ as a function of both colour and extinction. The "wavy" aspect of the top panel is a direct consequence of the third-order polynomial used for the modelling, where the need for the high-order had been tested by an ANOVA (see Sect. 4). The polynomial is well behaved in the interior of the fitting regime, but at the edges it generates a phenomenon termed "polynomial wiggle", whose main consequence is a lack of accuracy given by the large oscillations 

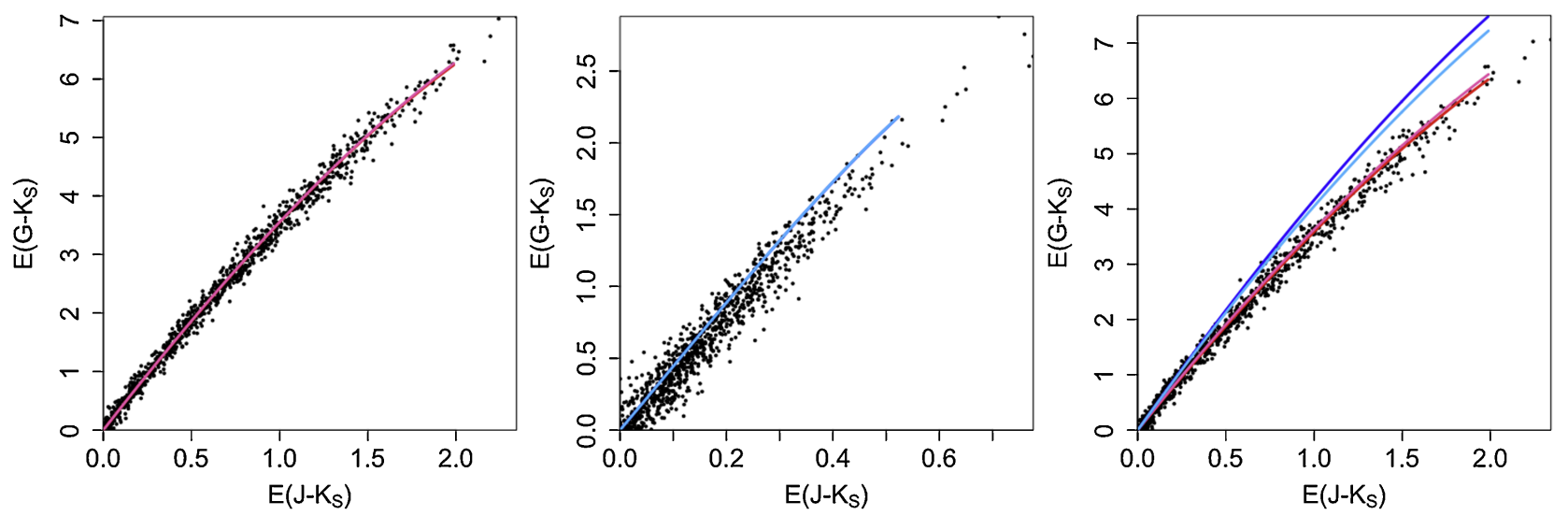

Fig. 4. Colour excesses $\mathrm{E}\left(G-K_{\mathrm{S}}\right)$ versus $\mathrm{E}\left(J-K_{\mathrm{S}}\right)$ for the red giants sample (left), the main sequence sample (center) and the combined sample (right). Black dots are the 1000 stars selected in each sample. Solid lines represent the colour excess increase with extinction for a reference temperature $T_{\text {eff }}\left(4136 \mathrm{~K}\right.$ for RG, red; $5550 \mathrm{~K}$ for MS, dark blue) and the corresponding reference colour $\left(G-K_{\mathrm{S}}\right)_{0}(2.49$ for RG, pink; 1.49 for MS, light blue). We note that some lines may overlap. Their cut corresponds to the interval of absorption $A_{0}$ indicated in Table 3 (13.3 for RG, 3.5 for MS).
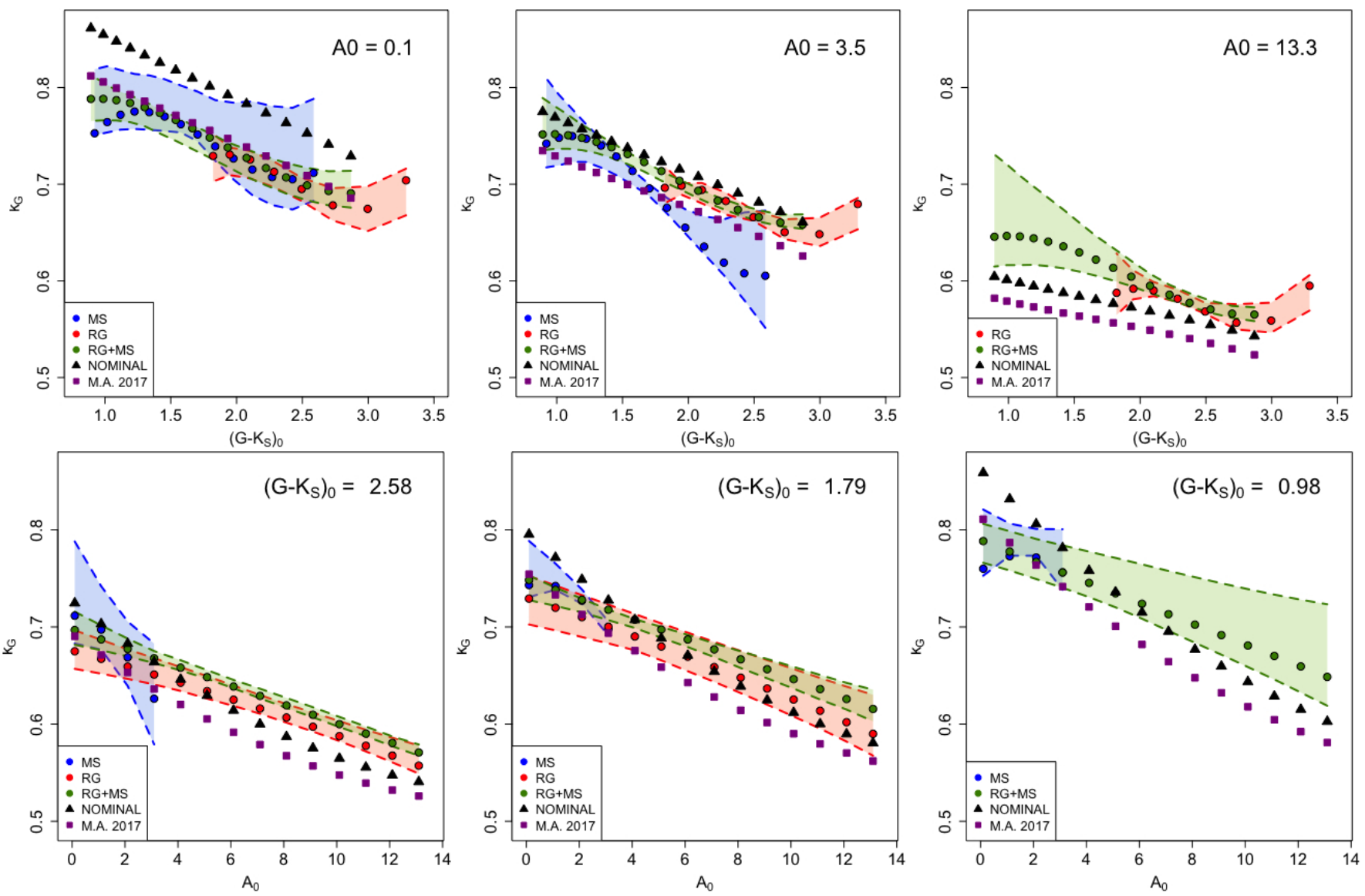

Fig. 5. Direct comparison of the empirical extinction coefficient $\mathrm{k}_{G}$ as a function of $\left(G-K_{\mathrm{S}}\right)_{0}$ for $A_{0}=0.1,3.5,13.3$ mag (top panels) and $A_{0}$ for $\left(G-K_{\mathrm{S}}\right)_{0}=2.58,1.79,0.98$ (bottom panels, which corresponds to $T_{\mathrm{eff}}=4020,5080,6500 \mathrm{~K}$ ). $A_{0}=3.5,13.3$ mag are the limit values for the MS and RG samples, respectively (see Table 3) while $\left(G-K_{\mathrm{S}}\right)_{0}=2.58,1.79$ are the maximal colour of LAMOST and the minimal colour of APOGEE, respectively. $\left(G-K_{\mathrm{S}}\right)_{0}=0.98$ is a sample case to show the behaviour at low colour indexes (i.e. high temperatures). Dots show the $\mathrm{k}_{G}$ mean value while the shaded area shows the $95 \%$ interval of confidence (see Sect. 5). Colours correspond to the red giants (RG, red), the main sequence (MS, blue) and unified sample (RG + MS, green). Black triangles and magenta squares show the theoretical $\mathrm{k}_{G}$ coefficient computed with the Gaia pre-launch (NOMINAL, Jordi et al. 2010) and Gaia G-DR1 (M.A. 2017, Maíz Apellániz 2017) passbands, respectively.

of the polynomial at both ends. For this reason, the accuracy is lower at the borders of the temperature and extinction $A_{0}$ intervals of validity (Fig. 5). For the extinction, the effect is less prominent as the polynomial is only of degree two in $A_{0}$.
However, its impact is seen in Fig. 5 (top panel, plot 2) where the $\mathrm{k}_{G}$ are not consistent with $\mathrm{RG}$ or $\mathrm{RG}+\mathrm{MS}$ due to this polynomial edge effect and lack of high-extinction stars in the MS sample. 
Table 3. Empirical extinction coefficient $\mathrm{k}_{G}$ for the Gaia $G$-band as a function of absorption $A_{0}$ and the normalised temperature $\hat{T}$ or colour $\left(G-K_{\mathrm{S}}\right)_{0}$ for the red giants sample (RG), the main sequence sample (MS) and both samples combined in only one (RG $\left.+\mathrm{MS}\right)$.

\begin{tabular}{|c|c|c|c|c|c|c|c|c|c|}
\hline$\underline{\mathrm{RG}}$ & $T_{\text {eff }}$ int. & $A_{0}$ & $a_{1}$ & $a_{2}$ & $a_{3}$ & $a_{4}$ & $a_{5}$ & $a_{6}$ & $a_{7}$ \\
\hline $\begin{array}{l}\mathrm{k}_{G}\left[\hat{T}, A_{0}\right] \\
\sigma_{k G}\left[\hat{T}, A_{0}\right]\end{array}$ & {$[3680,5080]$} & $<13.3$ & $\begin{array}{c}15.25 \\
3.69 \\
\end{array}$ & $\begin{array}{c}-51.059 \\
13.052\end{array}$ & $\begin{array}{l}59.12 \\
15.29 \\
\end{array}$ & $\begin{array}{c}-22.57 \\
5.94\end{array}$ & $\begin{array}{l}2.41 \mathrm{E}-03 \\
4.73 \mathrm{E}-03\end{array}$ & $\begin{array}{c}-1.42 \mathrm{E}-04 \\
9.01 \mathrm{E}-05\end{array}$ & $\begin{array}{c}-1.19 \mathrm{E}-02 \\
5.01 \mathrm{E}-03 \\
\end{array}$ \\
\hline $\begin{array}{l}\mathrm{k}_{G}\left[\left(G-K_{\mathrm{S}}\right)_{0}, A_{0}\right] \\
\sigma_{k G}\left[\left(G-K_{\mathrm{S}}\right)_{0}, A_{0}\right]\end{array}$ & $\frac{\overline{\left(G-K_{\mathrm{S}}\right)_{0} \text { int. }}}{[1.82,2.87]}$ & $\begin{array}{c}A_{0} \\
<13.3\end{array}$ & $\begin{array}{c}a_{1} \\
-0.72 \\
0.93\end{array}$ & $\begin{array}{c}a_{2} \\
1.94 \\
1.13\end{array}$ & $\begin{array}{c}a_{3} \\
-0.84 \\
0.45\end{array}$ & $\begin{array}{c}a_{4} \\
0.116 \\
0.059\end{array}$ & $\begin{array}{c}a_{5} \\
-1.24 \mathrm{E}-02 \\
2.83 \mathrm{E}-03\end{array}$ & $\begin{array}{c}a_{6} \\
-1.07 \mathrm{E}-04 \\
1.23 \mathrm{E}-04\end{array}$ & $\begin{array}{c}\frac{a_{7}}{1.68 \mathrm{E}-03} \\
1.14 \mathrm{E}-03\end{array}$ \\
\hline $\begin{array}{l}\mathrm{MS} \\
\mathrm{k}_{G}\left[\hat{T}, A_{0}\right] \\
\sigma_{k G}\left[\hat{T}, A_{0}\right]\end{array}$ & $\begin{array}{c}T_{\text {eff }} \text { int. } \\
{[4020,6620]}\end{array}$ & $\begin{array}{c}A_{0} \\
<3.5\end{array}$ & \begin{tabular}{c|}
$a_{1}$ \\
4.40 \\
1.38 \\
\end{tabular} & $\begin{array}{c}a_{2} \\
-11.405 \\
4.028\end{array}$ & $\begin{array}{c}a_{3} \\
11.52 \\
3.85 \\
\end{array}$ & $\begin{array}{c}a_{4} \\
-3.77 \\
1.21 \\
\end{array}$ & $\begin{array}{c}a_{5} \\
-0.051 \\
0.044 \\
\end{array}$ & $\begin{array}{c}a_{6} \\
-6.60 \mathrm{E}-03 \\
1.65 \mathrm{E}-03\end{array}$ & $\begin{array}{c}a_{7} \\
0.056 \\
0.038 \\
\end{array}$ \\
\hline $\begin{array}{l}\mathrm{k}_{G}\left[\left(G-K_{\mathrm{S}}\right)_{0}, A_{0}\right] \\
\sigma_{k G}\left[\left(G-K_{\mathrm{S}}\right)_{0}, A_{0}\right]\end{array}$ & $\frac{\overline{\left(G-K_{\mathrm{S}}\right)_{0} \text { int. }}}{[0.92,2.59]}$ & $\begin{array}{c}A_{0} \\
<3.5\end{array}$ & $\begin{array}{c}a_{1} \\
0.32 \\
0.15\end{array}$ & $\begin{array}{c}a_{2} \\
0.88 \\
0.30\end{array}$ & $\begin{array}{c}a_{3} \\
-0.53 \\
0.19\end{array}$ & $\begin{array}{c}a_{4} \\
0.097 \\
0.036\end{array}$ & $\begin{array}{c}a_{5} \\
0.038 \\
0.020\end{array}$ & $\begin{array}{c}a_{6} \\
-7.06 \mathrm{E}-03 \\
1.72 \mathrm{E}-03\end{array}$ & $\begin{array}{c}a_{7} \\
-0.017 \\
0.013\end{array}$ \\
\hline $\begin{array}{l}\mathrm{RG}+\mathrm{MS} \\
\mathrm{k}_{G}\left[\hat{T}, A_{0}\right] \\
\sigma_{k G}\left[\hat{T}, A_{0}\right]\end{array}$ & $\frac{T_{\text {eff }} \text { int. }}{[3680,6620]}$ & $\begin{array}{c}A_{0} \\
<13.3\end{array}$ & $\begin{array}{c}a_{1} \\
3.24 \\
0.55\end{array}$ & $\begin{array}{c}a_{2} \\
-8.31 \\
1.67\end{array}$ & $\begin{array}{c}a_{3} \\
8.72 \\
1.67 \\
\end{array}$ & $\begin{array}{c}a_{4} \\
-2.92 \\
0.55\end{array}$ & $\begin{array}{c}a_{5} \\
-7.55 \mathrm{E}-03 \\
6.24 \mathrm{E}-03 \\
\end{array}$ & $\begin{array}{c}a_{6} \\
-8.35 \mathrm{E}-05 \\
1.19 \mathrm{E}-04\end{array}$ & $\begin{array}{c}a_{7} \\
-6.73 \mathrm{E}-04 \\
6.73 \mathrm{E}-03\end{array}$ \\
\hline $\begin{array}{l}\mathrm{k}_{G}\left[\left(G-K_{\mathrm{S}}\right)_{0}, A_{0}\right] \\
\sigma_{k G}\left[\left(G-K_{\mathrm{S}}\right)_{0}, A_{0}\right]\end{array}$ & $\frac{\overline{\left(G-K_{\mathrm{S}}\right)_{0} \text { int. }}}{[0.92,2.87]}$ & $\begin{array}{c}A_{0} \\
<13.3\end{array}$ & $\begin{array}{c}a_{1} \\
0.697 \\
0.059\end{array}$ & $\begin{array}{c}a_{2} \\
0.219 \\
0.083\end{array}$ & $\begin{array}{c}a_{3} \\
-0.154 \\
0.037 \\
\end{array}$ & $\begin{array}{c}a_{4} \\
2.69 \mathrm{E}-02 \\
5.51 \mathrm{E}-03\end{array}$ & $\begin{array}{c}a_{5} \\
-1.14 \mathrm{E}-02 \\
3.43 \mathrm{E}-03\end{array}$ & $\begin{array}{c}a_{6} \\
-6.84 \mathrm{E}-07 \\
1.38 \mathrm{E}-04\end{array}$ & $\begin{array}{c}\frac{a_{7}}{6.58 \mathrm{E}-04} \\
1.30 \mathrm{E}-03\end{array}$ \\
\hline
\end{tabular}

Notes. For each sample we report temperature, colour, and extinction intervals of validity. The errors ( $1 \sigma$ uncertainties) on the coefficients have been measured with the bootstrap technique.

While there is a small difference between the empirically retrieved and the theoretical extinction coefficient (both nominal and G-DR1 (Maíz Apellániz 2017) passbands), the amplitude and the trend of the variation as a function of temperature (or colour) and extinction is similar. Our empirical coefficients are, as expected, closer to the G-DR1 passband than the nominal passband in the low-extinction regime. However they are larger than the theoretical ones for $A_{0}>3 \mathrm{mag}$ for the nominal passband, and $A_{0} \gtrsim 2 \mathrm{mag}$ for the $G$-DR1 passband. With the information currently in our possession we are not able to address this issue, which may be due to uncertainties in the extinction law or in the filter response determination. We will, however, perform the same study for the coming Gaia DR2 release in order to determine the DR2 $\mathrm{k}_{G}$ extinction coefficients and to clarify this problem.

Overall, we recommend the use of the combined sample (RG + MS) coefficients using the intrinsic colour $\left(G-K_{\mathrm{S}}\right)_{0}$. The use of the combined sample gives a unique solution for both stellar evolution stages, and it is less affected by the polynomial wiggle effect. The colour is also less affected by the temperature scale difference between LAMOST and APOGEE.

\section{Conclusions}

We present here the empirical estimation of the Gaia G-band extinction coefficient $\mathrm{k}_{G}$ that can be used as a unique solution for both red giants and main sequence stars.

We used high-quality photometry in the Gaia $G$-DR1 and 2MASS $J$ - and $K_{\mathrm{S}}$-bands combined with the APOGEE DR13 and the LAMOST DR2 spectroscopic surveys to retrieve effective temperatures for red giants and dwarfs samples, respectively. We implemented an MCMC method where we used the photometric calibration $T_{\text {eff }}$ versus $\left(G-K_{\mathrm{S}}\right)_{0}$ and $\left(J-K_{\mathrm{S}}\right)_{0}$ versus $\left(G-K_{\mathrm{S}}\right)_{0}$ relations (method presented by Ruiz-Dern et al. 2018), to estimate the extinction coefficient $\mathrm{k}_{G}$ as a function of the normalised temperature $\hat{T}=T_{\text {eff }} / 5040$ or colour $\left(G-K_{\mathrm{S}}\right)_{0}$ and absorption $A_{0}$.

We first compared each empirical $\mathrm{k}_{G}$ coefficient (measured for the dwarfs, the red giants, and the combined sample) with the theoretical coefficient (estimated using both the Gaia $G^{-}$ passband modelled pre-launch and the $G$-DR1 (Maíz Apellániz 2017) passband), and then we compared the empirical coefficients to one another. For the first case, while we find a small difference between our results and the theoretical extinction coefficients for large extinctions, we confirmed that both theoretical and empirical $\mathrm{k}_{G}$ have the same trend. For the second case we find consistent results.

We modelled the extinction coefficient as a function of both stellar temperature (or intrinsic colour) and absorption to more precisely account for the degeneracy between extinction and spectral energy distribution. We believe that this approach is the best practice, particularly for large passbands such as the Gaia Gband, where the extinction coefficient varies strongly within the band itself.

The results presented here are valid for the Gaia G-DR1 band data and they are constrained by the precision of the spectrometric data used for our analysis. The same study will be performed for the Gaia DR2 release (April 2018) with the inclusion of the estimation of the extinction coefficient valid for both BP and RP bands.

Acknowledgement. This work was supported by the Centre National détudes Spatiales (CNES) post-doctoral funding project. This work has made use of data from the European Space Agency (ESA) mission Gaia (https:// www.cosmos.esa.int/gaia), processed by the Gaia Data Processing and Analysis Consortium (DPAC, https://www.cosmos.esa.int/web/gaia/ dpac/consortium). Funding for the DPAC has been provided by national institutions, in particular the institutions participating in the Gaia Multilateral Agreement. This publication makes use of data products from the Two Micron All Sky Survey, which is a joint project of the University of Massachusetts and the Infrared Processing and Analysis Center/California Institute of Technology, funded by the National Aeronautics and Space Administration and the National 
Science Foundation. Funding for the Sloan Digital Sky Survey IV has been provided by the Alfred P. Sloan Foundation, the U.S. Department of Energy Office of Science, and the Participating Institutions. SDSS-IV acknowledges support and resources from the Center for High-Performance Computing at the University of Utah. The SDSS web site is www.sdss.org. SDSS-IV is managed by the Astrophysical Research Consortium for the Participating Institutions of the SDSS Collaboration including the Brazilian Participation Group, the Carnegie Institution for Science, Carnegie Mellon University, the Chilean Participation Group, the French Participation Group, Harvard-Smithsonian Center for Astrophysics, Instituto de Astrofísica de Canarias, The Johns Hopkins University, Kavli Institute for the Physics and Mathematics of the Universe (IPMU)/University of Tokyo, Lawrence Berkeley National Laboratory, Leibniz Institut für Astrophysik Potsdam (AIP), Max-Planck-Institut für Astronomie (MPIA Heidelberg), Max-Planck-Institut für Astrophysik (MPA Garching), Max-Planck-Institut für Extraterrestrische Physik (MPE), National Astronomical Observatories of China, New Mexico State University, New York University, University of Notre Dame, Observatário Nacional/MCTI, The Ohio State University, Pennsylvania State University, Shanghai Astronomical Observatory, United Kingdom Participation Group, Universidad Nacional Autónoma de México, University of Arizona, University of Colorado Boulder, University of Oxford, University of Portsmouth, University of Utah, University of Virginia, University of Washington, University of Wisconsin, Vanderbilt University, and Yale University. Guoshoujing Telescope (the Large Sky Area Multi-Object Fiber Spectroscopic Telescope LAMOST) is a National Major Scientific Project built by the Chinese Academy of Sciences. Funding for the project has been provided by the National Development and Reform Commission. LAMOST is operated and managed by the National Astronomical Observatories, Chinese Academy of Sciences.

\section{References}

Albareti, F. D., Prieto, C. A., \& Almeida, A. 2017, ApJS, 233, 25

Arenou, F., Luri, X., Babusiaux, C., et al. 2017, A\&A, 599, A50

Atwood, W. B., Abdo, A. A., Ackermann, M., et al. 2009, ApJ, 697, 1071

Bailer-Jones, C. A. L. 2010, MNRAS, 403, 96

Brooks, S., Gelman, A., Jones, G., \& Meng, X.-L. 2011, Handbook of Markov Chain Monte Carlo (CRC press)
Capitanio, L., Lallement, R., Vergely, J. L., Elyajouri, M., \& Monreal-Ibero, A. 2017, A\&A, 606, A65

Cardelli, J. A., Clayton, G. C., \& Mathis, J. S. 1989, ApJ, 345, 245

Castelli, F., \& Kurucz, R. L. 2003, in Modelling of Stellar Atmospheres, eds. N. Piskunov, W. W. Weiss, \& D. F. Gray, IAU Symp., 210, 20 Cohen, M., Wheaton, W. A., \& Megeath, S. T. 2003, AJ, 126, 1090 Draine, B. T. 2003, ARA\&A, 41, 241

Efron, B. 1987, J. Am. Stat. Assoc., 82, 171

Evans, D. W., Riello, M., De Angeli, F., et al. 2017, A\&A, 600, A51

Fitzpatrick, E. L., \& Massa, D. 2007, ApJ, 663, 320

Fitzpatrick, E. L., \& Massa, D. 2009, ApJ, 699, 1209

Gaia Collaboration (Brown, A. G. A., et al.) 2016, A\&A, 595, A2

Jordi, C., Gebran, M., Carrasco, J. M., et al. 2010, A\&A, 523, A48

Maíz Apellániz, J. 2017, A\&A, 608, L8

Marrese, P. M., Marinoni, S., Fabrizio, M., \& Giuffrida, G. 2017, A\&A, 607, A105

Martin, D. C., Fanson, J., Schiminovich, D., et al. 2005, ApJ, 619, L1

Mason, K. O., Breeveld, A., Much, R., et al. 2001, A\&A, 365, L36

Perryman, M. A. C., de Boer, K. S., Gilmore, G., et al. 2001, A\&A, 369, 339

Planck Collaboration I. 2011, A\&A, 536, A1

Plummer, M. 2003, JAGS: A program for analysis of Bayesian graphical models using Gibbs sampling

Rosen, S. R., Webb, N. A., Watson, M. G., et al. 2016, A\&A, 590, A1

Ruiz-Dern, L., Babusiaux, C., Arenou, F., Turon, C., \& Lallement, R. 2018, A\&A, 609, A116

Schlafly, E. F., Meisner, A. M., Stutz, A. M., et al. 2016, ApJ, 821, 78

Schlegel, D. J., Finkbeiner, D. P., \& Davis, M. 1998, ApJ, 500, 525

Skrutskie, M. F., Cutri, R. M., Stiening, R., et al. 2006, AJ, 131, 1163

Wu, Y., Du, B., Luo, A., Zhao, Y., \& Yuan, H. 2014, in Statistical Challenges in 21 st Century Cosmology, eds. A. Heavens, J.-L. Starck, \& A. Krone-Martins, IAU Symp., 306, 340

Xue, M., Jiang, B. W., Gao, J., et al. 2016, ApJS, 224, 23

York, D. G., Adelman, J., Anderson, Jr. J. E., et al. 2000, AJ, 120, 1579

Yuan, H. B., Liu, X. W., \& Xiang, M. S. 2013, MNRAS, 430, 2188

Zhao, G., Zhao, Y.-H., Chu, Y.-Q., Jing, Y.-P., \& Deng, L.-C. 2012, Res. Astron. Astrophys., 12, 723 